\title{
Diagnostic performance of a new red light LED device for approximal caries detection
}

\author{
Klaus W. Neuhaus • Philip Ciucchi • Jonas Almeida Rodrigues • \\ Isabelle Hug • Marta Emerich • Adrian Lussi
}

Received: 27 January 2014 / Accepted: 30 May 2014 / Published online: 7 June 2014

(C) Springer-Verlag London 2014

\begin{abstract}
The aim of this study was to test a newly developed LED-based fluorescence device for approximal caries detection in vitro. We assembled 120 extracted molars without frank cavitations or fillings pairwise in order to create contact areas. The teeth were independently assessed by two examiners using visual caries detection (International Caries Detection and Assessment System, ICDAS), bitewing radiography (BW), laser fluorescence (LFpen), and LED fluorescence (Midwest Caries I.D., MW). The measurements were repeated at least 1 week later. The diagnostic performance was calculated with Bayesian analyses. Post-test probabilities were calculated in order to judge the diagnostic performance of combined methods. Reliability analyses were performed using kappa statistics for nominal data and intraclass correlation (ICC) for absolute data. Histology served as the gold standard. Sensitivities/specificities at the enamel threshold were 0.33/0.84 for ICDAS, $0.23 / 0.86$ for BW, $0.47 / 0.78$ for LFpen, and $0.32 / 0.87$ for MW. Sensitivities/specificities at the dentine threshold were $0.04 / 0.89$ for ICDAS, $0.27 / 0.94$ for BW, 0.39/0.84 for LFpen, and 0.07/0.96 for MW. Reliability data were fair to moderate for MW and good for BW and LFpen. The combination of ICDAS and radiography yielded the best diagnostic performance (post-test probability of 0.73 at the dentine threshold). The newly developed LED device is not able to be recommended for approximal caries detection. There might be too much signal loss during signal
\end{abstract}

K. W. Neuhaus $(\varangle) \cdot$ P. Ciucchi $\cdot$ J. A. Rodrigues $\cdot$ I. Hug $•$

M. Emerich · A. Lussi

Department of Preventive, Restorative and Pediatric Dentistry,

School of Dental Medicine, University of Bern, Freiburgstrasse 7,

3010 Bern, Switzerland

e-mail: klaus.neuhaus@zmk.unibe.ch

\section{J. A. Rodrigues}

Department of Pediatric Dentistry, School of Dentistry, Federal

University of Rio Grande do Sul, Porto Alegre, Brazil transduction from the occlusal aspect to the proximal lesion site and the reverse.

Keywords Caries detection · Caries diagnostics $\cdot$ Laser fluorescence $\cdot$ Radiography $\cdot$ ICDAS

\section{Introduction}

Approximal caries detection is usually performed with visualtactile inspection in combination with radiography [1]. However, visual inspection of proximal surfaces is hampered by the neighboring teeth. While the features to describe caries as defined by the International Caries Detection and Assessment System (ICDAS) are theoretically applicable to approximal smooth surfaces [2], the limited visibility of the respective surfaces usually requires additional detection aids. Due to its simplicity of practice and the fact that it allows for instantaneous decision making, radiography is "the most widespread lesion detection aid" [1]. If dentine involvement is regarded as the threshold for invasive intervention, radiography has advantages because it detects more dentine caries than the naked eye [3]. If the threshold is shifted to include enamel lesions as well, there is no advantage because, in lowcaries-prevalence populations, the number of false-positive findings outweighs the number of additionally detected dentine lesions. Thus, concerns have been raised that bitewing radiography (BW), in addition to visual inspection, in lowcaries-prevalence populations leads to unacceptably high rates of false-positive findings, thus initiating unnecessary invasive treatment [4]. Furthermore, because radiography uses ionizing radiation and exposes patients to a small but detectable risk to becoming harmed, it was suggested that "alternative methods to using ionising radiation in caries diagnosis should be considered once their diagnostic validity has been clearly established" [5]. 
The fiber-optic transillumination (FOTI) of teeth, which works fine for slim front teeth, was found to be inferior to radiography. While specificities of proximal caries detection were high, the sensitivity of FOTI was significantly lower than that for bitewing radiography [6]. It was concluded that FOTI cannot replace bitewing radiography. There is no clinical study that has validated quantitative light-induced fluorescence (QLF) in approximal caries lesions. Thus, at the time of writing this report, the laser fluorescence (LF) method using a pen-type device (LFpen) has been shown to offer the most advantages for approximal caries detection [7, 8]. A recent controlled clinical trial showed that the ability to detect dentine lesions is comparable to bitewing radiography [9]. However, LFpen's minimum dimension of $0.4 \mathrm{~mm}$ is still too big to assess all interproximal areas and correctly measure caries beneath the contact area.

Recently, a LED-based pen-type device has been developed (Midwest Caries I.D., MW) to detect both occlusal and approximal caries. In contrast to LFpen, the manufacturers purport that lesion detection for any kind of lesions can be performed occlusally. The LED light is claimed to penetrate deeper into the tooth than the LFpen red laser light; thus, the backscattered fluorescence signal of proximal lesions is supposed to be captured from the occlusal aspect above the marginal rim. For occlusal lesions, this device showed promising sensitivity of 0.7 and specificity of 0.9 on the D3 level [10].

The aim of this in vitro study was to validate approximal caries detection by the newly developed LED-based device and compare it to visual inspection with ICDAS, bitewing radiography, and the established LFpen method.

\section{Materials and methods}

Tooth selection and sample preparation

A sample of 120 permanent molars and premolars was selected from a pool of frozen extracted teeth. The frozen teeth had been stored at $-20^{\circ} \mathrm{C}$ since extraction [11]. The selected teeth were either visually caries free or had enamel and dentin caries on their approximal surfaces. All the teeth were cleaned with a toothbrush (Trisa ultra-super-sensitive, Trisa AG, Triengen, Switzerland) under tap water for $15 \mathrm{~s}$ to remove plaque. The teeth were then placed in an ultrasonic bath (Cavitron, Dentsply, Konstanz, Germany) for $15 \mathrm{~s}$ in order to eliminate calculus and debris.

All teeth were numbered and stored individually in $100 \%$ humidity. Photographs of the approximal surface were taken with a microscope (Leica M420 DC300, Leica, Heerbrugg, Switzerland) at a magnification of $\times 6.25$. One spot per surface was chosen to be detected and was marked on the printout of the photograph.
The teeth were randomly sorted in pairs and placed in contact with each other in order to create an approximal contact point and corresponding interproximal space. The roots of each pair of teeth were then embedded in self-curing resin (Paladur, Hereaus, Hanau, Germany) with the crowns remaining exposed. Gingival tissue was mimicked using a medium-consistency polyvinylsiloxane (GI-Mask Automix, Colténe-Whaledent, Altstätten, Switzerland).

\section{Caries detection procedures}

Two independent and trained clinicians examined each pair of assembled, embedded teeth twice with a 7-day interval. The examiners were blinded as to presence or absence of lesions on the approximal surface of the teeth. The approximal lesions were also visually inspected and classified using the ICDAS scoring system [12]:

0 - sound tooth

1 - first visual change in dry enamel

2 - distinct change in moist enamel

3-localized enamel breakdown

4 - underlying dark shadow from dentine

5 - distinct cavity with visible dentine

6 - extensive distinct cavity with visible dentine

Radiographic examinations were carried out on bitewings of each pair taken with a dental radiograph (HDX DentalEZ, Malvern, PA, USA) with milliamperes at 0.16 for $0.09 \mathrm{~s}$. The lesions on the radiographs were classified using a scoring system [8]:

0 - no radiolucency visible

1 -radiolucency visible in enamel

2 -radiolucency visible in dentine restricted to the outer third of dentine

3 -radiolucency visible in dentine extending to the middle third of dentine

4-radiolucency visible in dentine extending to the inner third of dentine

The lesions were also tested using LFpen (DIAGNOdent pen, KaVo, Biberach, Germany) with an approximal tip according to the manufacturer's instructions and classified using cutoff values [7]:

$$
\begin{aligned}
& \text { D0- }(0-6) \\
& \text { D1- }(6.1-13) \\
& \text { D2- }(13.1-17) \\
& \text { D3, D4-(>17) }
\end{aligned}
$$

The lesions were finally tested using a LED-based device (Midwest Caries I.D., Dentsply, Des Plaines, IL, USA) 
according to the manufacturer's instructions and classified according to audible signal frequencies:

0 - no caries (green light, no beeping noise)

1 - enamel caries (red light, slow beeping noise)

2 - superficial dentine caries (red light, moderate beeping noise)

3 - deep dentine caries (red light, fast beeping noise)

\section{Histology}

The pairs of teeth were then separated using a diamond disc (Isomet 11-1180 low speed saw, BUEHLER LTD, Lake Bluff, IL, USA; $101.67 \mathrm{~mm}$ diameter, $0.3 \mathrm{~mm}$ thickness). Each tooth was then ground longitudinally up to $1 \mathrm{~mm}$ before the measurement site on a Knuth Rotor polishing machine (Struers, Ballerup, Denmark) with silicone carbide paper of $60 \mu \mathrm{m}$ grain size under cooled tap water. The progression of the grinding process was constantly checked under a microscope (Leica) at a magnification of $\times 6.25$. When the periphery of the site was reached, silicone carbide paper with a grain size of 30 , 18,8 , and $5 \mu \mathrm{m}$, respectively, was used. Photographs of the ground site were taken at $\times 6.25$ using a microscope (Leica). The cut surfaces were cleaned and dehydrated in solutions of increasing alcohol concentration, with the addition of basic $0.5 \%$ fuchsine (Inselspital-Apotheke, Bern, Switzerland) for staining. The surfaces were then photographed at $\times 10$ (Leica) and scored by an experienced third examiner, who was otherwise not involved in the study, using the following classification [13]:

$$
\begin{aligned}
& \text { D0 - no caries } \\
& \text { D1 — caries extending to the outer half of the enamel } \\
& \text { D2 - caries extending to the inner half of the enamel } \\
& \text { D3 - caries extending to the outer half of the dentine } \\
& \text { D4 - caries extending to the inner half of the dentine }
\end{aligned}
$$

Histology served as the gold standard for further statistics.

\section{Statistics}

The software program SAS (Cary, NC, USA) was used. To allow for statistical comparisons of the obtained results, the scores were collapsed in order to discriminate sound, enamel caries, and dentine caries. For ICDAS, the $\mathrm{ICDAS}_{3}$ criteria were applied: sound (0), enamel caries $[1,2]$, and dentine caries [3-6]. A reliability analysis was carried out using weighted kappa statistics for scored outcomes and intraclass correlations for concrete LFpen outcomes.

Sensitivity, specificity, and combined post-test probabilities were calculated for all methods. The level of significance was set at 0.05 for all tests.
Table 1 Bayesian analysis

\begin{tabular}{lllll}
\hline & LFpen & MW & BW & ICDAS \\
\hline Sensitivity & D1 & & & \\
Specificity & 0.47 & 0.32 & 0.23 & 0.33 \\
PPV & 0.82 & 0.87 & 0.86 & 0.84 \\
& D3 & 0.84 & 0.80 & 0.82 \\
Sensitivity & 0.39 & 0.07 & 0.27 & 0.04 \\
Specificity & 0.84 & 0.96 & 0.94 & 0.89 \\
PPV & 0.51 & 0.42 & 0.65 & 0.61 \\
\hline
\end{tabular}

\section{Results}

The distribution of caries was as follows: D0, 38; D1, 31; D2, 14; D3, 22; and D4, 13. Two teeth were lost during sample processing.

The calculated combined sensitivity and specificity values are presented in Table 1. For dentine caries, the combined post-test probabilities of ICDAS (0.61) and BW resulted in diagnostic improvement (0.73), while additional use of LFpen showed no diagnostic gain (0.6), and the combination with MW resulted in diagnostic loss (0.51) (Table 2).

The intra-examiner reliability analysis showed moderate to good agreement for LF and BW. However, MW and ICDAS showed unacceptably low levels of agreement below 0.5 (Table 3). Inter-examiner reliabilities were moderate to good for LF, BW, and ICDAS, but weak for MW (0.431) (Table 3).

Cross-tabulations showed that all methods suffered from large false-negative proportions in this study. MW diagnosed enamel caries correctly in 5/45 cases (11\%) and dentine caries in $1 / 35$ cases $(3 \%)$ (Table 4$)$.

\section{Discussion}

The idea to assess proximal caries occlusally above the marginal rim is intriguing because known problems with interproximal accessibility of diagnostic devices can be avoided. However, the newly developed LED-based caries detection device failed to support visual proximal caries detection.

Table 2 Post-test probabilities

\begin{tabular}{llc}
\hline & D1 & D3 \\
\hline ICDAS & Post-test probability & \\
& 0.81 & 0.61 \\
ICDAS $\times$ LFpen & Combined post-test probability \\
ICDAS $\times$ MW & 0.63 & 0.60 \\
ICDAS $\times$ BW & 0.67 & 0.51 \\
& 0.61 & 0.73 \\
\hline
\end{tabular}


Table 3 Reliability analysis

\begin{tabular}{lll}
\hline & Method & Degree of agreement \\
\hline Intra-examiner reliability & Ex 1 LFpen & 0.69 (ICC) \\
& Ex 2 LFpen & 0.84 (ICC) \\
& Ex 1 MW & 0.23 (kappa) \\
& Ex 2 MW & 0.50 (kappa) \\
& Ex 1 BW & 0.68 (kappa) \\
& Ex 2 BW & 0.73 (kappa) \\
& Ex 1 ICDAS & 0.40 (kappa) \\
& Ex 2 ICDAS & 0.43 (kappa) \\
Inter-examiner reliability & LFpen & 0.86 (ICC) \\
& MW & 0.43 (kappa) \\
& BW & 0.66 (kappa) \\
& ICDAS & 0.64 (kappa) \\
\hline
\end{tabular}

While the established methods showed moderate to good agreement, the Midwest device produced unacceptably low numbers of inter- and intra-examiner agreement.

The intra-examiner agreement for the ICDAS scores was approximately 0.4 for both examiners. This is considerably lower than the value of 0.65 that is required by the ICDAS group [12]. However, this number is achievable in directly accessible surfaces only [2], whereas our results reflect the difficulties and pitfalls of visual inspection on proximal surfaces with tooth contacts. The inter-examiner reliability detected for LFpen (ICC 0.86) is slightly better than the data reported by Novaes et al. [14] (ICC 0.75). This could be expected because the latter study was a clinical study, where usually the values that can be obtained in optimal in vitro conditions cannot be achieved.

The cross-tabulations clearly showed a strong trend towards false-negative findings for all methods (Table 4). Low values could be expected for ICDAS because the questionable caries detection sites were simply not visually accessible. Some improvement of visual diagnostics would be expected by temporary tooth separation (e.g., with orthodontic separation rubbers), but this procedure is time-consuming and therefore is not routinely used clinically. However, a sensitivity of 0.27 was found on the dentine caries level for radiography. One reason might be that, in the tooth selection of the present study, teeth with frank cavitations were excluded. Thus, the dentine caries may have been rather superficial dentine caries, where radiographic detection might have been more difficult than in medium to deep dentine lesions. In absolute numbers, LFpen had the largest proportion of correct diagnoses.

The tested LED device produced low numbers for sensitivity $(0.32$ at the enamel threshold, 0.07 at the dentine threshold, respectively) while maintaining high specificity $(0.87$ at the enamel threshold, 0.96 at the dentine threshold). A weakness in detecting enamel caries could be anticipated because a sensitivity of 0.31 for occlusal lesions was described [10]. The sensitivity to detect dentine caries was much lower than the value of 0.7 reported for occlusal lesions [10]. An explanation could be that the backscattered fluorescence signal from the approximal lesion is weakened too much when traveling to the relatively large amount of healthy tooth substance beneath the marginal rim. Deflection, scattering, and absorption are known phenomena that hamper light signal transduction through teeth [15]. Another reason could be that the distance between the approximal lesion and measuring point is much larger than in occlusal lesions. While light intensity diminishes exponentially with growing distance by natural law, further signal loss can be expected by reflection, scattering, and absorption.

Regarding the obtained values of sensitivities and specificities, higher values have been reported previously. Lussi et al. [7] calculated sensitivities $>0.8$ both at the enamel and dentine thresholds. The findings in this study demonstrated sensitivities of 0.47 for the enamel threshold and 0.39 for the dentine threshold. We suggest that, in the present study setup, the use of a gingival mask might have interfered with optimum readings because it restricted the accessibility of the approximal area for the LFpen tip. This is in line with the in vivo findings of Novaes et al. [14] who reported a sensitivity of 0.16 at the enamel threshold and 0.55 at the dentine threshold. However, as mentioned earlier, frank cavitations were excluded in the present study. Nevertheless, the gingival mask used in the present in vitro study should not have influenced the LED readings.

Additional diagnostic devices have often been recommended as a "second opinion" in the decision-making process [16]. In order to calculate the combined effect of two different methods, the positive likelihood ratio can be used to calculate the post-test odds of a test, which then become the pretest odds for a second independent test with a known $\mathrm{LR}+$, resulting in
Table 4 Cross-tabulations with gold standard

LFpen cutoffs: $0,0-7 ; 1,7.1-17$; $2,>17$. MW cutoffs: 0 , green light; 1 , slow beeping; 2 , moderate to fast beeping

0 sound, 1 enamel caries, 2 dentine caries

\begin{tabular}{|c|c|c|c|c|c|c|c|c|c|c|c|c|c|c|}
\hline & & \multicolumn{3}{|c|}{ ICDAS } & \multicolumn{3}{|c|}{ BW } & \multicolumn{3}{|c|}{ LFpen } & \multicolumn{3}{|c|}{ MW } & \multirow[t]{2}{*}{$\Sigma$} \\
\hline & & 0 & 1 & 2 & 0 & 1 & 2 & 0 & 1 & 2 & 0 & 1 & 2 & \\
\hline \multirow[t]{3}{*}{ Histology } & 0 & 30 & 5 & 3 & 34 & 1 & 3 & 32 & 3 & 3 & 33 & 4 & 1 & 38 \\
\hline & 1 & 35 & 4 & 6 & 40 & 3 & 2 & 31 & 5 & 9 & 39 & 5 & 1 & 45 \\
\hline & 2 & 21 & 4 & 10 & 20 & 5 & 10 & 14 & 8 & 13 & 20 & 14 & 1 & 35 \\
\hline$\Sigma$ & & 86 & 13 & 19 & 94 & 8 & 15 & 77 & 16 & 25 & 92 & 23 & 3 & 118 \\
\hline
\end{tabular}


the post-test probability of their combination $[17,8,18,19]$. Our results show that the "classic" combination of visual caries detection with bitewing radiography results in the best achievable diagnostic accuracy (Table 4). In contrast, the use of the LED fluorescence device resulted in considerably less desirable diagnostic performance than visual inspection alone.

The LED fluorescence device gives both optical and acoustic feedback. It appeared in our study and in pretests that several examiners, one of which is an accomplished musician, could not clearly discriminate between the moderate and fast beeping noise. For the results of the present study, this is of little significance because the scores for dentine caries (red light) were collapsed in the statistical analysis. However, it would be easier to have four different colors rather than three different beeping noises, of which two were hardly discriminable.

\section{Conclusion}

Midwest Caries I.D. in its current form is not able to be recommended. Although the idea of measuring approximal caries from the occlusal aspect is appealing, signal reflection, absorption, and scattering might hamper optimum readings.

Acknowledgments We would like to thank Walter Bürgin for carrying out the statistical analysis of this study.

\section{References}

1. Neuhaus KW, Ellwood R, Lussi A, Pitts NB (2009) Traditional lesion detection aids. Monogr Oral Sci 21:42-51

2. Ekstrand KR, Luna LE, Promisiero L, Cortes A, Cuevas S, Reyes JF, Torres CE, Martignon S (2011) The reliability and accuracy of two methods for proximal caries detection and depth on directly visible proximal surfaces: an in vitro study. Caries Res 45:93-99

3. Machiulskiene V, Nyvad B, Baelum V (2004) Comparison of diagnostic yields of clinical and radiographic caries examinations in children of different age. Eur J Paediatr Dent 5:157-162
4. Baelum V (2010) What is an appropriate caries diagnosis? Acta Odontol Scand 68:65-79

5. European Commission (2004) Radiation protection 136-European guidelines on radiation protection in dental radiology

6. Vaarkamp J, ten Bosch JJ, Verdonschot EH, Bronkhoorst EM (2000) The real performance of bitewing radiography and fiber-optic transillumination in approximal caries diagnosis. J Dent Res 79:17471751

7. Lussi A, Hack A, Hug I, Heckenberger H, Megert B, Stich H (2006) Detection of approximal caries with a new laser fluorescence device. Caries Res 40:97-103

8. Rodrigues JA, Diniz MB, Josgrilberg EB, Cordeiro RC (2009) In vitro comparison of laser fluorescence performance with visual examination for detection of occlusal caries in permanent and primary molars. Lasers Med Sci 24:501-506

9. Huth KC, Lussi A, Gygax M, Thum M, Crispin A, Paschos E, Hickel R, Neuhaus KW (2010) In vivo performance of a laser fluorescence device for the approximal detection of caries in permanent molars. J Dent 38:1019-1026

10. Rodrigues JA, Hug I, Neuhaus KW, Lussi A (2011) Light-emitting diode and laser fluorescence-based devices in detecting occlusal caries. J Biomed Opt 16:107003

11. Francescut P, Zimmerli B, Lussi A (2006) Influence of different storage methods on laser fluorescence values: a two-year study. Caries Res 40:181-185

12. Topping GV, Pitts NB (2009) Clinical visual caries detection. Monogr Oral Sci 21:15-41

13. Downer MC (1975) Concurrent validity of an epidemiological diagnostic system for caries with the histological appearance of extracted teeth as validating criterion. Caries Res 9:231-246

14. Novaes TF, Matos R, Braga MM, Imparato JC, Raggio DP, Mendes FM (2009) Performance of a pen-type laser fluorescence device and conventional methods in detecting approximal caries lesions in primary teeth-in vivo study. Caries Res $43: 36-42$

15. Karlsson L (2010) Caries detection methods based on changes in optical properties between healthy and carious tissue. Int J Dent 2010:270729

16. Lussi A, Hibst R, Paulus R (2004) DIAGNOdent: an optical method for caries detection. J Dent Res 83(Spec No C):C80-C83

17. Lussi A, Firestone A, Schoenberg V, Hotz P, Stich H (1995) In vivo diagnosis of fissure caries using a new electrical resistance monitor. Caries Res 29:81-87

18. Rodrigues JA, Hug I, Diniz MB, Lussi A (2008) Performance of fluorescence methods, radiographic examination and ICDAS II on occlusal surfaces in vitro. Caries Res 42:297-304

19. Neuhaus KW, Rodrigues JA, Hug I, Stich H, Lussi A (2011) Performance of laser fluorescence devices, visual and radiographic examination for the detection of occlusal caries in primary molars. Clin Oral Investig 15:635-641 